\title{
Communication and Distribution Management System in SCADA
}

\author{
Mrunmayee Kore ${ }^{1}$, Mohini Chaube ${ }^{2}$, Gaurang $\operatorname{Singh}^{3}$, Shruti Nema ${ }^{4}$ \\ B.E. Student, Department of Electrical Engineering, Lokmanya Tilak College of Engineering, Navi Mumbai, India ${ }^{1,2,3}$ \\ Professor, Department of Electrical Engineering, Lokmanya Tilak College of Engineering, Navi Mumbai, India ${ }^{4}$
}

\begin{abstract}
In this paper, various aspects of communication techniques in SCADA is discussed. The integration of the communication system along with the entire distribution network is very crucial for real-time, up to date information. This benefits the personnel to take quick action in case of fault occurrence. This paper also deals with how the Distribution Management System (DMS) improves reliability in the system and forms an important component of the entire distribution network.
\end{abstract}

Keywords: SCADA, DMS, MPLS, Communication System

\section{INTRODUCTION}

Due to the increasing advancements in information and technology, electrical utilities are strengthening their distribution of power using automation. Among the various automation techniques, SCADA is one of the most costeffective solutions. Supervisory Control And Data Acquisition (SCADA) in itself means it is a software that supervises and controls the hardware i.e. Remote Terminal Unit (RTU) and also acquires data from these devices through a communication system as shown in fig 1 .

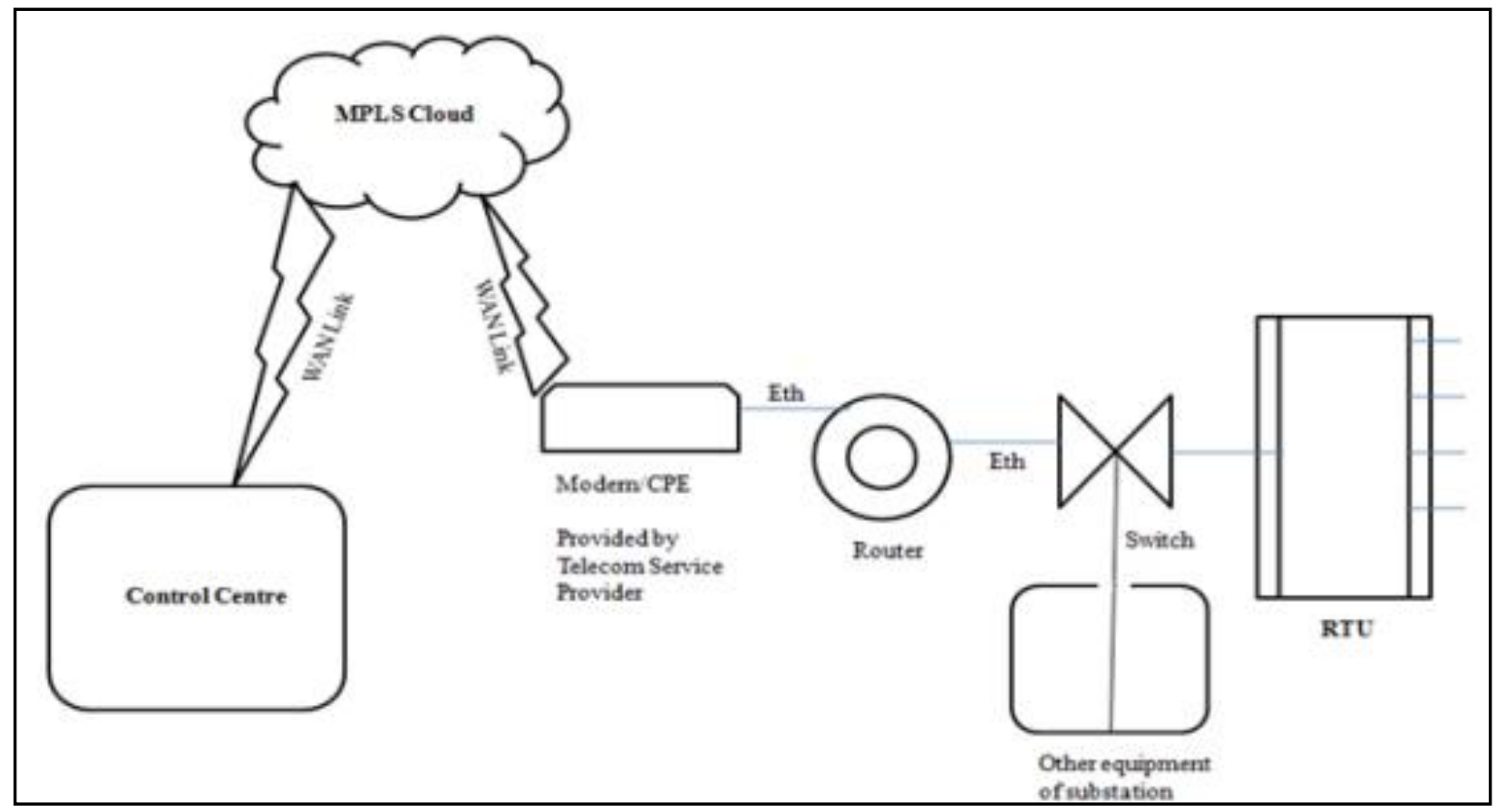

Fig1. Communication System

The communication takes place through a WAN link which is obtained by integrating various LAN networks. The interfacing techniques used in MPLS wherein short path packets are generated and are provided with labels which are more secure and reliable than earlier used technique like Time Division Multiplexing (TDM). MPLS uses end to end encryption to provide security to the system wherein it converts the data into the codes. The next element that is modern is an internet access provider that is connected to a router which is used for connecting various devices to a network through Ethernet cable. 


\section{COMMUNICATION SYSTEM OF SCADA}

2.1 Multiprotocol Label Switching (MPLS): MPLS is an acronym for Multi-Protocol Label Switching. It is a technique utilized in high-performance telecommunication networks. Labels are assigned to data packets consisting source and destination information such as IP addresses, bandwidth, etc and labels are used to carry data from node to node rather than long network addresses. This is beneficial as it reduces the time required to transmit the data packet by avoiding complex lookups. MPLS is believed to lie between the data link layer and network layer which are Layer 2 and Layer 3 respectively. It is Layer 2.5 protocol. It supports different traffics such as Asynchronous Transfer Mode (ATM), Frame Relay, Synchronous Optical Networking (SONET) and Ethernet. It belongs to the family of packaged switch networks but is designed to be used on both packet-switched networks and circuit-switched networks.

\section{Advantages:}

1. Scalability: It facilitates the addition of remote and close sites without the significant burden of hardware requirement. It can be used on a small scale like offices, among buildings or large distance communication.

2. WAN routing: WAN routing can be handled by the service provider which makes it hassle-free for the user

3. Quality of Service: MPLS is accompanied by QoS like jitter buffer, traffic shaping which is provided by the service provider in order to meet the traffic requirements and avoid degradation.

4. Service Level Agreements (SLAs): SLAs are the foundational agreements between customer and service provider which consists responsibilities of both parties. Response time and resolution time are two of the set of metric which forms the basis of evaluation of service provider's performance includes customer's requirements specified and penalties incurred on breaking the norms.

2.2 General Packet Radio Services (GPRS): General Packet Radio Services (GPRS) 171.2 Kbps. It enables communication through cellular networks. It is a $2.5 \mathrm{G}$ network. It utilizes GSM infrastructure and hence provides higher data rates on an already existing 2G GSM network. It utilizes packet technology switching, unlike GSM which uses circuit switching. The data is broken into various packets that travel along the most efficient path and reach provided and reassembles at the destination using the header address. GPRS is assigned 1 to 8 dynamic time slots on the basis of coding Standards namely CS-1, CS-2, CS-3, and CS-4. As the number of time slots progresses along with the Coding Standard, the data rate increases proportionally. $172 \mathrm{kbps}$ is the highest for CS-4 standard with 8 slots. The advantages of GPRS are as follows:

1. Packet-based service: GPRS being packet-based service enables faster transfer of data than circuit-based switching and it utilizes network bandwidth to complete the transmission.

2. Cost-Efficient: GPRS charges data based on the amount of data transferred in contrast to GSM and other circuit switching technology which charges based on the length and duration of the connection.

3. Versatile Application Support: GPRS supports various forms of application transfer like images, videos, voice mails, SMS (Short Message Service) on cell phones and desktop computers.

4. Security: It safe and secure transfer of the data, confidentiality, and protection from external attack.

2.3 CDMA: CDMA, an acronym for Code-Division Multiple Access is a channel access method that allows multiple users to make use of the same channel. This optimizes the use of that particular channel. It operates in the frequency range of $800 \mathrm{MHz}$ to $1.9 \mathrm{GHz}$. It digitizes the incoming data into binary data with a specific code. The data is scattered across the available bandwidth and are reassembled at the receiving end with the help of the unique assigned code. In this way, there are multiple coded data in a spectrum and they are carried together which makes sure the spectrum is utilized to its optimum. Despite different data, the coding ensures increased security and reduces the cross talk.

2.4 Ethernet: Ethernet is Local Area Network (LAN) connecting technology. It is utilized in small spaces like buildings or rooms. Institute of Electrical and Electronics Engineers Inc. (IEEE) specifies Ethernet in the family of standards called IEEE 802.3. With the help of specific protocols and hardware, multiple computers and other hardware are connected. Ethernet cards are connected to PCs and Ethernet cables connect multiple computers together. It has two units of transmission namely a frame which consists of the payload of data and address of MAC (Media Access Control) of the sender as well as a receiver.

2.5 Global Positioning System (GPS): GPS stands for Global Positioning System and consists of space segment i.e.24 GPS satellites deployed in 6 orbits with 4 in each orbit. They revolve around the Earth at a height of $20,000 \mathrm{~km}$. The second segment is the control segment which refers to the ground control stations which monitors and keeps track of satellite and make sure they do not deviate along their axis and the timing beyond their tolerance level. The third segment is the user segment which is the receivers of the data like planes, people using maps to track a location, ships, 
Vol. 8, Issue 2, February 2019

addresses of the locations, etc. The GPS satellite sends the time signal to a specific point. The difference between the time the GPS receiver receives the signal and the GPS time is measured. This procedure is repeated with the remaining three satellites. This helps in determining the distance of the GPS receiver from the GPS receiver to the three satellites. The atomic clock gives accurate data in comparison to the clock installed in the GPS satellite. The fourth satellite helps in estimating the distance of receiver compared to the other three satellites thus reducing the error margin.GPS satellites has 2 operational frequencies L1 (1,575.42 MHz) and L2 (1,227.60 MHz). The orbital data of each satellite is known as ephemeris and gives the accurate satellite location for positioning information. The orbital data of all satellites is known as almanac and has orbit and status information of all the satellites.

2.6 IEC 60870-5-103: IEC (International Electro-technical Commission) set a standard named IEC 60870-5-103. It was devised for a power system, their communication with protective equipment and control systems. It uses either Application Service Data Units (ASDU) or generic services for transmission of data. It supports both master and slave initiated messages for data transfer. The data is divided into various information object and each one has a specific address. It has the ability to prioritize the data.

2.7 Multi-Function Transducer (MFT): Multi-Function Transducer is a versatile product and can be used by all possible combinations for LT/HT application and 3 phase/ 3 wire/4 wire configuration. It measures voltage, current, power factor, frequency, active, real and reactive power. This can be used for 4 quadrant power. If given $2 / 3$ current outputs equivalent to the measured basic electrical quantities or panel quantities through MODBUS communication. The MFT is configurable for all kinds of combinations of power measurements. Both analog and digital communication interfaces enable accurate and reliable electrical measurement and communication. It has 4 analog inputs and two digital outputs and has a response time of less than $300 \mathrm{msec}$.

\section{How the System Works with all elements:}

A fault occurs on the network at the point indicated on the diagram by the 'FAULT' arrow. Circuit breaker C11 trips as soon as the fault is detected. All customers between C11 and switch S16 are now off the supply. Telecontrol alarms arrive at the control room via existing SCADA; engineers know that C11 has TRIPPED. There is no further information. A slow process of switching sections of the network in and out is the only way to identify the location of the fault. Engineers are severely restricted in what they can do to speed restoration and reduce further disruption to customers. The location of the fault will involve repeatedly closing onto the faulty feeder in order to cause trips (or not). This unnecessarily stresses the plant and may lead to secondary faults.

All iHost screens displaying information about the feeder now show F11, F12, and F13 as reporting the alarm. Users can clearly identify where the fault is. Visualization of the feeder is available through iHost Mimic display of network in and out is the only way to identify the location of the fault. Fig2 shows how a network functions with all the elements connected. FPI units between C11 and the FAULT detect the passage of fault current and communicate with iHost using 'report by exception' messages.

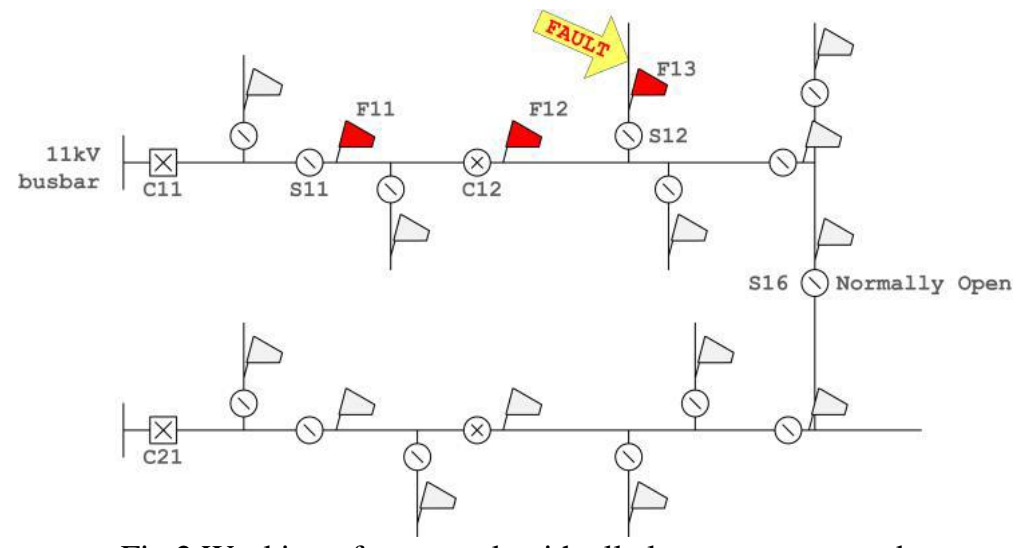

Fig 2.Working of a network with all elements connected

FPI alarms arrive from locations F11, F12 and F13. Other FPIs does not detect the fault current and do not report alarms. iHost immediately updates the 'Virtual RTU' which represents all the monitored points on the feeder above.DMS/NMS systems continuously poll iHost for new alarms. Control room displays are updated to show the location of the fault as beyond F13. Control engineers instruct field staff to open switch S12; then remotely close C11. All customers on healthy network sections are back on supply in the shortest possible time with the minimum number of switching operations. 


\section{DISTRIBUTION MANAGEMENT SYSTEM (DMS)}

Distribution Management System (DMS) integrates field equipment, communication devices, control centre to create a centralized operation centre and enables efficient management to increase benefits. It helps in reducing complexities and improves reliability. It improves control room operation and key performance indicators. In order to make optimal use of energy resources dynamic decisions are becoming necessary and help personnel in monitoring, deciding and implementing various actions.

\section{Operation without DMS:}

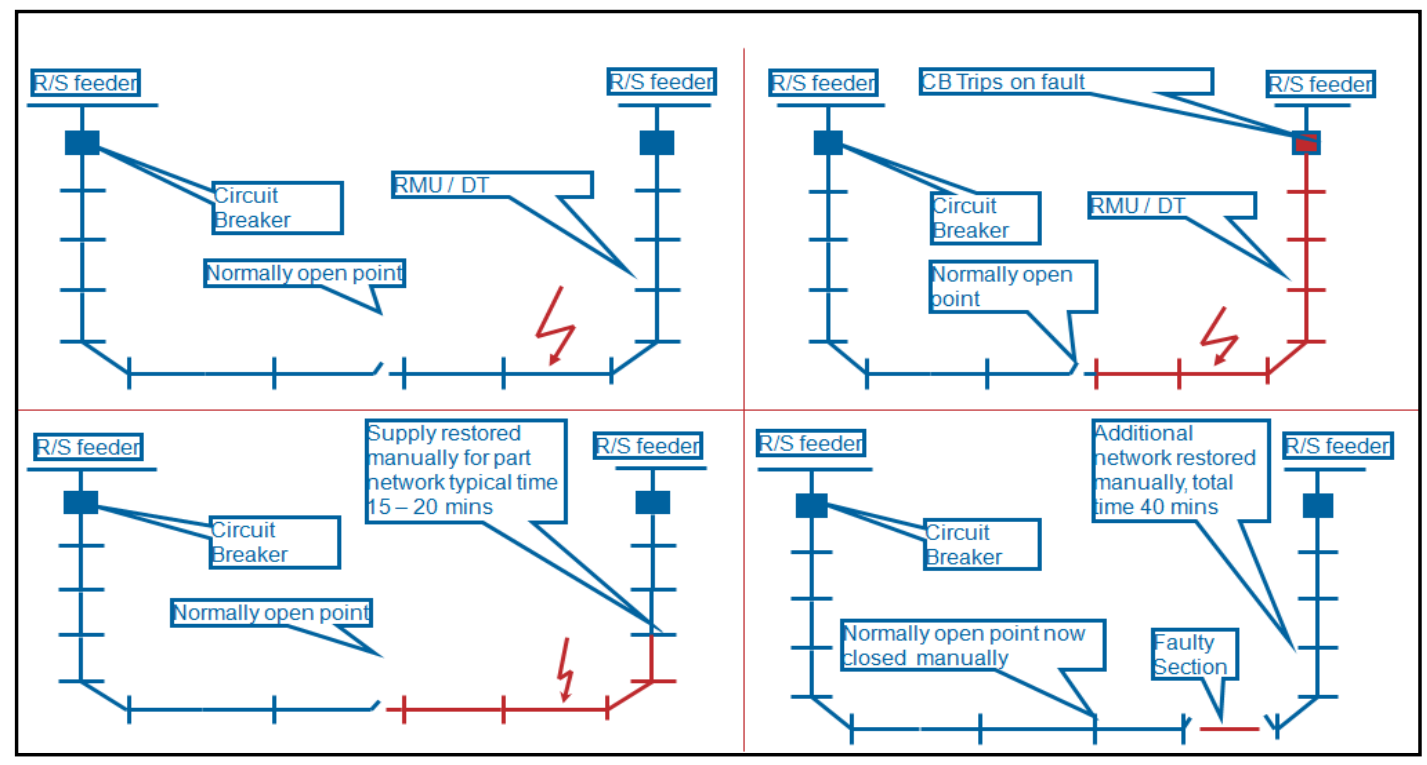

Fig3.Operation without DMS

Fig 3 represents the operation without DMS. When a fault occurs in a system, the current goes on increasing which eventually operates the circuit breaker. The healthy part and faulty parts are separated from each other. In the absence of DMS, the line maintenance has to check every feeder from the circuit breaker to the location of a fault. Supply restoration takes about 20 minutes. This causes wastage of time of the consumer and the staff workers during fault identification and proves uneconomical for the distribution centre.

\section{DMS operation:}

1. The Open ring network:

The open-ring network of switching substations has a single input through two circuit-breakers. For efficient fault detection, one ring main unit (RMU) with an open switch must be placed somewhere in the ring network. In order to achieve fast, efficient and fast localization of fault, there should be at least one indicator in every RMU. Most of the indicators are retrofitted which makes it easy to expand and add new indicators anytime.

2. Fault situation in an open-ring network:

When a fault is in one of the cables between the circuit-breaker and the ring main units, the indicators measure the fault current. All the indicators between the circuit-breaker and the fault location will trigger and indicate a fault passage. In order to avoid any further damage to the cables and the ring main units, the circuit-breaker cuts the supply to the complete line along which the fault has occurred. The fault location is eased by the use of DMS as the fault occurs between the last triggered indicator and the first non-triggered indicator. The defective cable will be cut off from the rest of the ring.

3. The restored situation of an open ring network:

After isolating the faulty cable, the open ring network can be re-energized. This ensures that the staff gets enough time to repair the cable and the time of power outage to the consumer is also reduced considerably. The triggered indicators will reset in standby mode by time reset, manual reset, by recovering operating current or recovering operating voltage of the monitored network. The normal service situation is now restored. 
Vol. 8, Issue 2, February 2019

Figure 4.shows the operation with DMS. With the use of DMS provided with RMU and fault passage indicator (FPI), on the occurrence of the fault, the circuit breaker operates. FPI detects the fault in the fault zone.

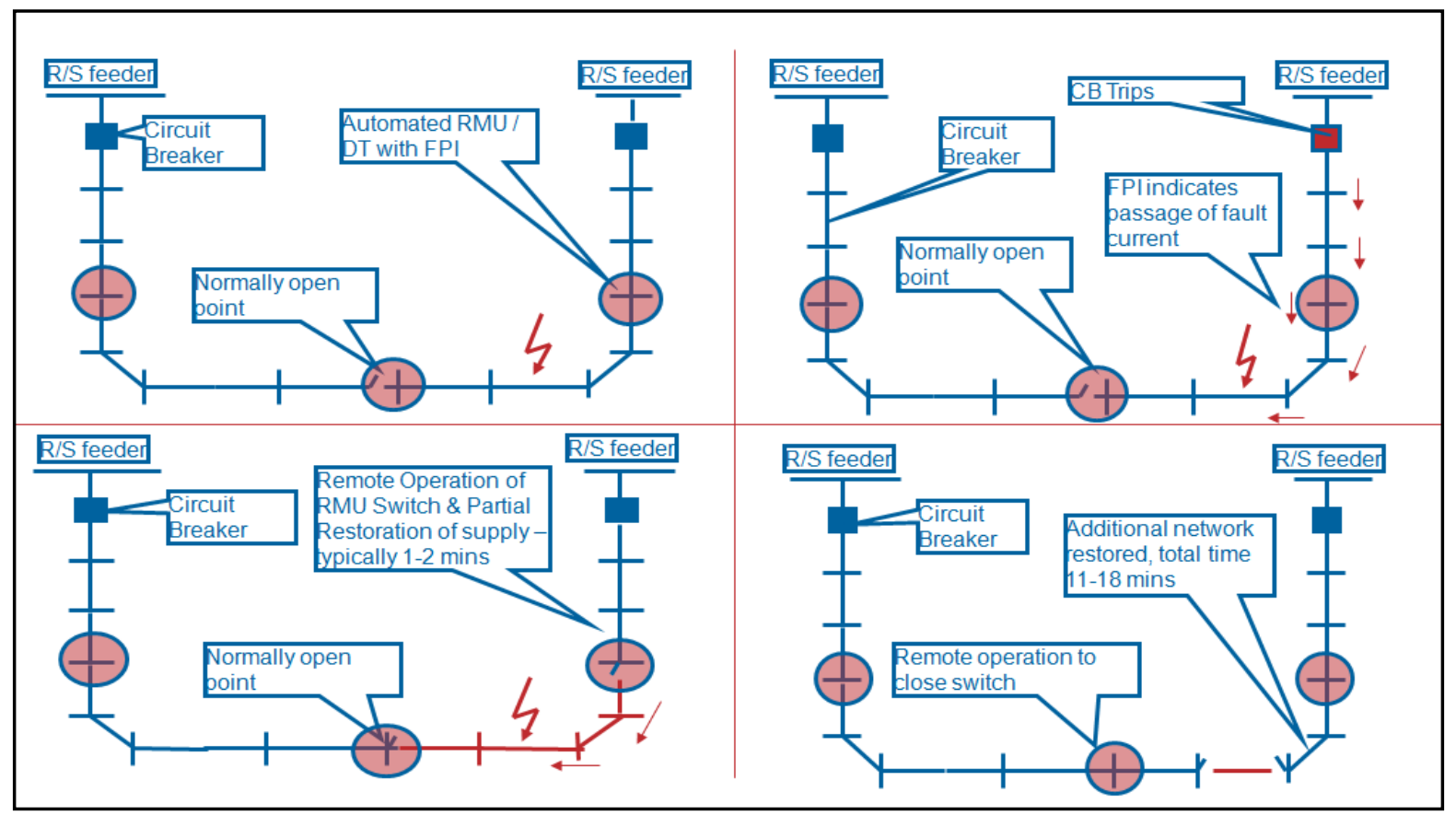

Fig 4.Operation with DMS

The supply continuity is regained in the zone between circuit breaker to particular FPI from another section of RMU (typically within 1-2 min).

\section{Benefits of DMS:}

Connectivity: DMS allows communication through cellular devices and receives data electronically directly from the field, which helps the staff to constantly be informed about the on-field operations.

Distribution Network Analysis: It helps to determine the change in voltage and frequency and eases the voltage calculations and provides optimum switching guidance.

Scalability: As the system is expanded after a certain duration due to an increase in load demand, DMS can easily be integrated with the new system Without much hassle, DMS can be made to perform all the functions for the new system as that for the old system.

\section{CONCLUSION}

SCADA is a system that helps to acquire dynamic data and keep track of the up to date performance of each component and communication system is an integral part of it. Various components are connected together and each of them performs a specific function by gathering data and over a wide expansion transmitting them to a centralized control centre. DMS improves the efficiency, reduces financial losses that would have incurred in its absence and make it possible to determine the exact location of fault which reduces further damage.

\section{REFERENCES}

[1]. https://searchnetworking.techtarget.com/tip/MPLS-advantages-and-disadvantages-for-WAN-connectivity

[2]. https://searchitchannel.techtarget.com/definition/service-level-agreement

[3]. https://www.youtube.com/watch?v=qmoP6UQHhWw

[4]. https://www.elprocus.com/cdma-technology-working-applications/

[5]. https://www.furuno.com/en/gnss/technical/tec_what_gps

[6]. https://w3.usa.siemens.com/smartgrid/us/en/webinars/leadthecharge/Documents/DistributionMgmtSystem_Whitepaper.pd 